\title{
A Case study on Automatic Smart Headlight System for Accident Avoidance
}

\author{
RD. Balaji ${ }^{a}$ \\ "College of Applied Sciences- Salalah, Sultanate of Oman. \\ * Corresponding Author: balaji.sal@cas.edu.om
}

Received : $22^{\text {th }}$ January 2020, Accepted : 30 March 2020

\begin{abstract}
The recent developments in the sensors and the IoT (Internet of Things) technologies are transforming many of the devices smart and to act independently or with minimum human intervention. The researchers are identifying many existing systems and trying to address the current problems in the functionalities or limitation of these existing devices. The researchers are also working to discover the technologies which are very expensive and not in the reach of the common man so that it would be possible to bring out the better solution for the ever-growing mankind with suitable affordability.
\end{abstract}

The functionalities of IoT are not limited to a specific field and outweigh the other traditional methodologies; it is being used in many of the life saving applications in recent days including health care, emergency care, accident-aids and many more. The recent survey reveals that the accidents percentage is high between evening 6 to morning 6 compared to the day time. The top most reason for these accidents are temporary blindness of the drivers caused by the headlights of the opposite side vehicles. Another study shows that the accident chances are high during raining or foggy season than a normal day. To avoid these accidents, we are mainly depending on the fog lights. There are few technologies already existing with the luxury cars but these are highly expensive to be adopted in the economy cars.

This paper proposes the technology which can be used to make the headlight system to be smart to avoid many existing reasons for the night time or bad weather accidents on road. The researchers' main objective is to design an economic Automatic Smart Headlight system which can be used by the automobile industry. The second main objective is to design the headlight system for the two and three wheelers also, which is not actually considered by many of the researchers or the automobile industries.

Keywords: IoT, Smart Headlight, Microcontroller, Arduino Board, Embedded System, Sensors. 


\section{Introduction}

In developing and developed nations the Governments are giving higher priority for the road infrastructures. They consider the road infrastructure will boost their economy in many ways and it is a true fact too. The automobile industry also improved their vehicles in many aspects, mainly speed of the vehicles. The accidents on roads are having very high adverse effects compared to olden days due to modern vehicles with the provision of high speed. Many studies throughout the world have proved that the accident percentage is high during night time compared to the day time. It is not less common that accidents are high at day time as well especially during bad weather like rainy day, foggy morning or evening and snowy time. There are many factors playing major role during these accidents, but the top most reason for these accidents are poor visibility of the drivers [1].

The light comes from the vehicles which are coming on the opposite direction blinds the drivers for few seconds. Again the driver can see things clearly only after few seconds of getting the normal light. The roads which are not straight and having many curves are also having many blind spots which lead to accidents in the hilly areas or on the roads having many curves [2].

The drivers are paying much attention to change the high beam or low beam or vice versa. This creates lot of problems to the drivers who are driving on the opposite direction. There are few luxury cars having semi smart lights which automatically changes the high beam to low beam whenever required. But this technology is not adopted by the economy car models.

The economy cars head lamps can be made smarter than as on today. Most of the cars and the two wheelers are having many lights like head light, indicator lights, fog lights etc., When the number of lights are more it is difficult for the drivers to control all these whenever it is required. Hence this paper tries to design a single automatic smart head light system which can address almost all the needs of the head lights in the front and back of the car.

Even during the normal condition, we don't have any system to indicate that there is a vehicle in the blind spot. Few modified side mirrors are really helpful but these mirrors need the physical attention of the driver and has no indicators.

No much studies have conducted for the two and three wheelers by the researchers and almost all these problems are existing for the drivers who drive the two/three wheelers. Hence this research study proposes Automatic Headlight Systems for these vehicles also. Hence the researchers give importance for the economical solutions for these problems.

The researchers are trying to design automatic headlight system which will automatically respond to the climate and the environment. At the same time, it also indicates the driver about the existing climate and environment details and the current status of the headlight. 
This paper lists few of the sensors and the micro controller which can be used to design such a system. In the design section the researchers are giving the headlight system design and the whole system. The researchers also give design of the backside lighting systems and the blind spot vehicle designing systems. The next section discusses the current and relevant studies and systems about the Smart Headlights.

\section{Literature Review}

The automatic head light system research and implementation are happening from the late 90's. The automatic or adaptive head lights are used in the luxury cars like BMW, Mercedes, Volvo, Audi etc., but the sensors and other components used in these cars are very expensive [3].

One of the reviewed approaches has an orientation control system for the headlights which actually rotates the right and the left low beams independently and makes the beam parallel to the curved roads as much as possible for the better visibility in the curved, narrow or mountain roads [4]. In these kinds of systems, they have used two systems, one is for the vehicle and road and the second system is the actual head light system. The second system takes the input from the first system and based on the details it does few calculations and prepares commands based on these calculations and sends to the motor connected with the head light.

There are few systems proposed by the researchers which actually adjusting automatically the direction of optical axes of the headlight using the spherical sensors comprised of metal balls surrounded by the fluid encapsulated with the spherical sensors and also connected to the spherical sensor system [5]. This system is also using the computer controlled unit, which is kept behind the headlight very closely. Hence the metal ball cooperates with the sensor which is part of the spherical sensor system. This will make the head light function along with the car movement direction.

In another research, researchers proposed an Electric Control Unit (ECU) system for the headlights of the cars or trucks which gets the outputs of the sensors arrangement that is supplied to the above specified electric control unit using a bus for the communication purpose. In this research, the system calculates the value of a radius of the vehicles when it turns on the road which is used further for changing the direction of the optical axes of the swivel lights. The values are computed based on the output of the sensors which are mentioned above. These values go to the ECU and the calculations give the value to adjust the optical axis [6]. The ECU actually calculates the optical axis angle to be controlled using the normal values calculated in ECU. These values are used to activate the actuators which plays a major role in changing the direction of the optical axis of the headlights.

In a research paper called "Adaptive Front Light System" [7] the researcher proposed a headlight control system which is similar to the previous one. The researcher proposes a headlight system which swings horizontally based on the movement of the steering angle and the 
also swings vertically based on the distance of the opposite car approaching this vehicle. The researcher gave at most importance for the consistency, accuracy, and the availability of the components used in the adaptive front light system. Researcher also considered the total cost of the system and try to build a cost effective adaptive head light system. The objective is also extended to improve the visibility of the drivers of the vehicles to achieve the safety of the people in the vehicle. This system is also takes the input from the sensors. The sensors sensed values will be passed to the FPGA (Field-Programmable Gate Array) which is the head of the system. This head of the system is connected to the motor which moves the headlights horizontally or vertically. The sensors proposed by the researchers are ultrasonic distance sensor and potentiometer. The second sensor used for measuring the steering rotation angle. The first sensor helps to make the vertical movement of the headlights whereas the second sensor helps to decide the movement of the headlights horizontally. Similar to other researchers, in this research also they aim to make the mechanical movement of the headlight for the better visibility.

The next section lists out some of the sensor nodes needed for the design of the proposed system, which is then followed.

\section{Components of the Proposed System}

LDR (Light Dependent Resister - Light Detecting Sensors): Sensor for measuring the day and night light.

Doppler Radar Sensor (Pulsar): Sensor for measuring rain.

OFS (Optical Fog Sensor): Sensor for measuring the fog.

VIP (Video Image Processor) with imaging sensor [8]: Sensor for identifying the cars on the right and left side of the vehicles.

Acceleration Detectors: Sensors for measuring the steering rotation and speed.

Ultrasonic Sensors and PIR (Passive Infrared) sensors: Sensors for identifying the opposite vehicles distance.

ILD (Inductive Loop Detectors), Magnetometers: Sensors for identifying the side track of the road.

Arduino UNO (R3) Board: Consists of a micro controller for the program to activate the part of the lighting system based on the input from the sensors. This type of board is cheaper as well.

As per the main objective of this research we have selected the components which are cost effective, so that this system can be adopted in the sector of economic cars or automobile vehicles (two wheelers, three wheelers, cars, trucks or medium size vehicles). The possible facilities that can be given and automated to the cars and two wheelers are shown in the figure 3.1 . 


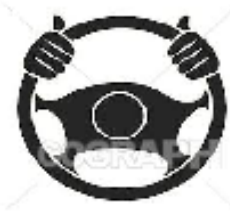

Steering controller

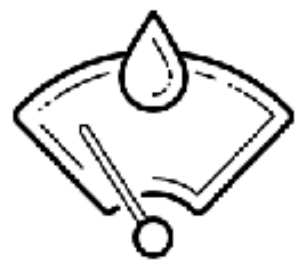

Rainfall indicator
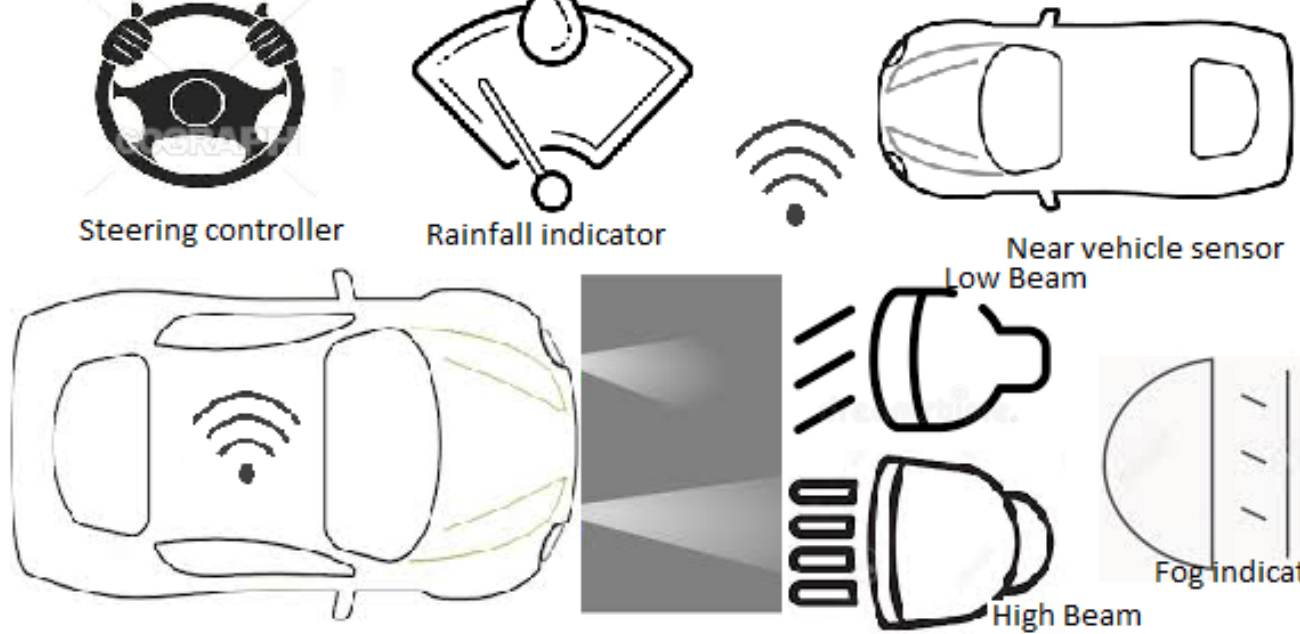

Near vehicle sensor

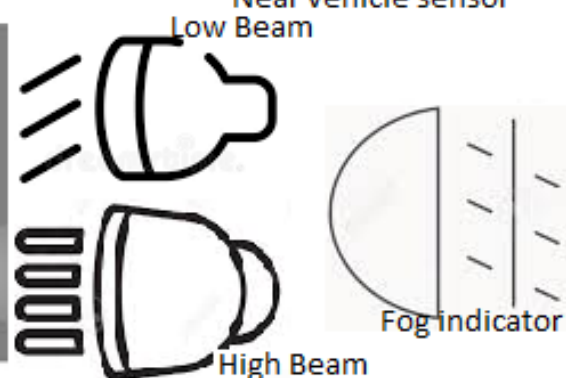

Fig. 3.1 Automated controls needed for a vehicle

\section{Proposed System Design}

This paper concentrates on proposing an integrated automatic lighting system for the automobile vehicles. This system can be adapted to any type of vehicles with the minimum change in the micro controller program. For the experiment setup the researchers have decided to use Arduino board as the electronics platform which includes a micro controller. All the sensors which we have decided to use are mentioned in the previous section.

In our work, we have decided to avoid any mechanical movement in the lighting system. Instead we have introduced many parts in the lighting system dedicated for different environment which are explained in this section.

Using the LDR, the system identifies the intensity of the light outside the vehicle and either switch ON and OFF the headlight system. Usually the outer LED border lights will be on first, when it becomes darker, the board makes the dim or low beam light ON. When the distance measuring sensors show there are no vehicles opposite to this vehicle then high beam will be ON and other lights will be OFF. To make this system intelligent the sensors on the top of the vehicle find whether the vehicle is in the open space or within a building. If it is within the building high beam headlight will not be $\mathrm{ON}$ even when there is no vehicle in the opposite direction.

The ultrasonic sensors will be used to find the vehicles opposite to the vehicle. If there is a vehicle nearby then the vehicle, then the high beam will be switched to the low beam light. If 
the vehicle is quite far from the vehicle, then the high beam light will be on with the dark layer in the middle. Otherwise the complete high beam headlight system will be ON.

When the rain and fog sensors give the positive signal then the fog light and the tail fog lights will be $\mathrm{ON}$ which will help the vehicles that come behind this vehicle. This system can be improved by connecting the wiper system. When it is raining, the wiper will also be $\mathrm{ON}$ by the same controller. The speed can be decided based on the intensity of the rainfall.

The side object identifying sensors helps to inform the driver about the vehicles in the blind spot and make the side light ON. The side lights will be ON when the vehicle steering rotated more than certain angle by the driver. This motion will automatically switch on the side indicators based on the rotation direction, if it is not activated by the driver. If the driver makes the side indicator $\mathrm{ON}$, then the side lamp of that side will be $\mathrm{ON}$ as well. In the more sophisticated cars the cameras of that side will be activated and display for the information of the drivers.

When the vehicle goes close to the track lines, the information will be displayed on the monitor for the driver's information with a beep sound. If this is at night time, then the side lights will be $\mathrm{ON}$ and $\mathrm{OFF}$ continuously.

This is the system proposed by the researchers. Further the researchers will work on a simulation and the programming part of the controller using Arduino board. The proposed simple design of the circuit is in figure 4.1.

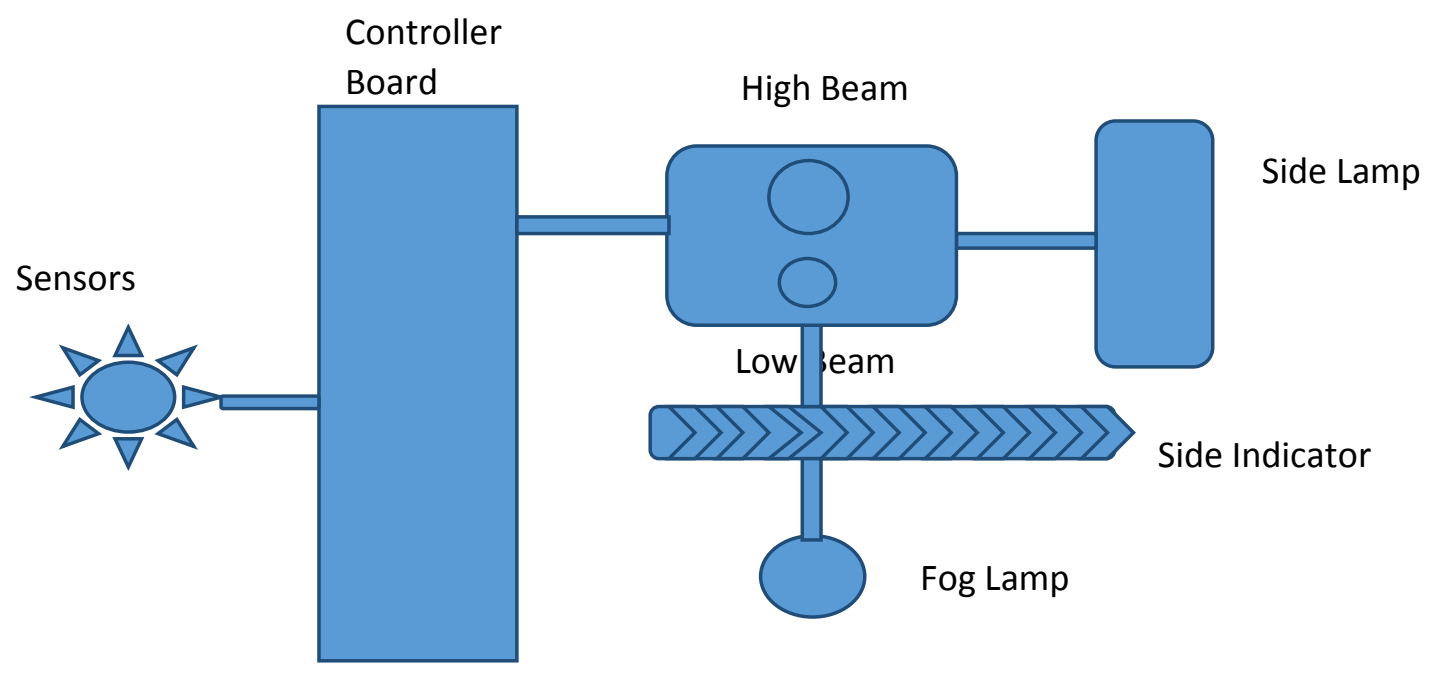

Fig 4.1 Circuit Diagram 


\section{Conclusion}

The above proposed model and the components will be the one stop solution for all the problems related to the head lights either at the night time or in the adverse weather. The components proposed are also very cheap compared to the existing components in the luxury cars like BMW, Mercedes, Audi etc., Hence this can be adapted in the economy sector cars. The cost of the sensors may be comparatively higher which are available in the market for the professional use compared to the sensors which we are using for the research purpose. The proposed circuit diagram is using only one head light, fog light, indicator light and side light since this can also be used for the two wheelers. Whereas for the cars, three more light sets to be connected with the controller board and the program will be bit more complicated compared to the two wheeler system. In the upcoming research two different set of models to be designed and simulated for cars and the two wheelers. The system can be extended by sharing the information to the driver via the infotainment system in the car. The wired connection can be converted to the wireless connection using ZigBee or any other wireless technology for the communication between the sensors and the control board, as well as the control board to the lighting system. The previous researchers have worked with the automatic headlight system which moves the headlight mechanically. But this proposed system presenting two unique system models which can be used for the two wheelers as well as the headlight system without mechanical movement.

\section{References}

[1] S. K. Choudhary., R. Suman., Sonali., H. Banga., "Electronic Head Lamp Glare Management System for Automobile Applications", International Journal of Research in Advent Technology, Vol. 2, No. 5, pp. 402-416, 2014.

[2] S. Shreyas, K. Raghuraman, A. P. Padmavathy, S. A. Prasad, G. Devaradjane, "Adaptive Headlight System for Accident Prevention”, International Conference on Recent Trends in Information Technology, April 2014.

[3] T. Hacıbekir, S. Karaman, E. Kural, E. S. Öztürk, M. Demirci and B. A. Güvenç, "Adaptive Headlight System Design Using Hardware-In- The-Loop Simulation”, Automotive Control and Mechatronics Research Center, Proceedings of the 2006 IEEE International Conference on Control Applications, Munich, Germany, October 4-6, 2006.

[4] J. Youn, M. D. Yin, J. Cho, D. Park, "Steering Wheel-based Adaptive Headlight Controller with Symmetric Angle Sensor Compensator for Functional Safety Requirement”, 2015

[5] J. De, "Universal Adaptive Headlight System", IEEE International Conference on Vehicular Electronics and Safety, Hyderabad, India, December 16-17, 2014.

[6] S. Shashikant, M. A. Mechkul, "Adaptive front Light System" IOSR Journal of Electronics and Communication Engineering, 2019. 
[7] https://vtechworks.lib.vt.edu>APPENDIX-BPDF.pdf, Accessed on 14.1.2020.

\section{Acknowledgements}

The author declare that they have no conflict of interest.

\section{Conflict of interest}

The author declare that they have no conflict of interest.

\section{About The License}

(C) 2020 The Authors. This work is licensed under a Creative Commons Attribution 4.0 International License which permits unrestricted use, provided the original author and source are credited. 